\title{
Nucléaires civil et militaire : des recherches liées
}

Jacques Bordé, physicien, retraité du CNRS, et Michèle Leduc, physicienne, CNRS

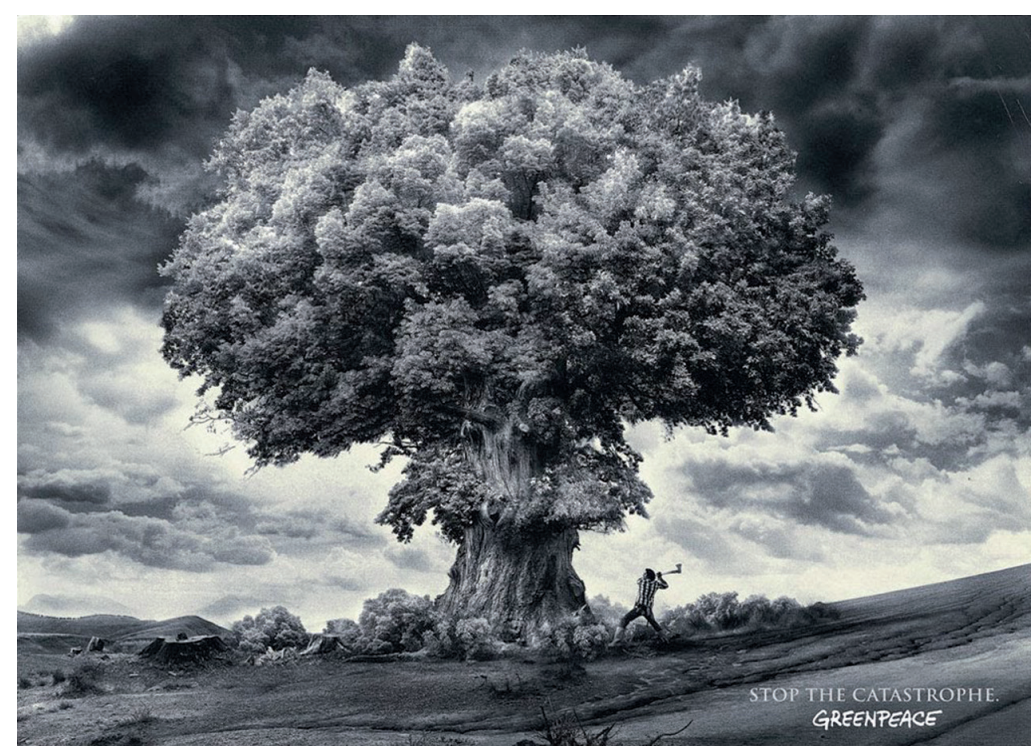

\section{Le développement du nucléaire en} France est le fruit de choix politiques qui ont établi un lien étroit entre la recherche militaire et celle sur les applications civiles. Les conséquences de ce lien sont multiples et ont conduit les physiciens à s'interroger sur leur rôle.
Le développement du nucléaire civil et celui du nucléaire militaire en France sont intimement liés. Dès l'origine, en particulier à travers toute l'histoire du CEA, l'étude de la physique nucléaire s'est développée dans l'interaction constante entre la recherche fondamentale et les applications industrielles, civiles et militaires ${ }^{(a)}$. Encore aujourd'hui, une partie de la recherche nucléaire civile s'attaque à des problèmes fondamentaux dont la solution intéresse les militaires pour améliorer, diversifier et mieux contrôler leur arsenal nucléaire. De même, la recherche militaire sur l'armement nucléaire est considérée comme duale par les décideurs politiques, c'est-à-dire qu'elle devrait avoir des retombées pour la recherche civile, pas seulement nucléaire, et pour l'industrie en général (surtout de l'armement, mais pas seulement). À ce titre, l'État donne un budget conséquent aux militaires, justifié devant les citoyens en affirmant qu'une partie sert à la recherche civile, et tire notre industrie vers le haut avec une technologique d'excellence ${ }^{(b)}$. C'est le sens de deux rapports parlementaires récents $[1,2]$.

\section{Des croisements multiples}

Outre la connaissance approfondie des noyaux et des réactions nucléaires, de nombreux domaines de recherche sont communs au nucléaire militaire et au nucléaire civil. Citons par exemple la séparation isotopique, le traitement des déchets et le démantèlement des équipements, les questions de sécurité et de cyber-sécurité, la pérennisation de l'approvisionnement en combustibles, la miniaturisation des composants, la compréhension de la sismologie, etc... La médecine nucléaire a longtemps bénéficié des équipements militaires mieux dotés, notamment pour la fourniture en produits radioactifs à partir d'uranium enrichi de qualité militaire. De même, les militaires ont bénéficié de l'expertise des médecins nucléaires pour la radioprotection des soldats et la santé publique dans les zones d'essais nucléaires. Aujourd'hui il y a une justification à la coopération dans ce domaine médical, à cause de l'impact des bombes dites sales et des armes à uranium appauvri. 
Il y a toujours eu des croisements de connaissances et des allers-retours d'innovation entre les applications civiles et militaires du nucléaire. Ainsi, il est probable que le quasi-doublement du budget (de 3,5 à 6 milliards d'euros par an) en discussion pour la dissuasion nucléaire, à cause du démantèlement et de la modernisation des sous-marins nucléaires lanceurs d'engins (SNLE), aura des retombées sur la recherche et l'industrie civile. Le rapport parlementaire de 2016 [1] détaille bien la dualité industrielle dans de nombreux secteurs, par exemple pour la balistique, avec le parallèle entre le programme Ariane et le missile M51.

\section{L'exemple du programme «Simulation »}

Pour illustrer la dualité avec le monde de la recherche universitaire, prenons l'exemple du programme "Simulation". Depuis que la France s'est engagée à ne plus provoquer d'explosions nucléaires en signant le traité d'interdiction complète des essais nucléaires (TICEN), elle met au point l'amélioration de ses équipements par la simulation ${ }^{(c)}$. Elle s'est dotée de calculateurs de puissance, le Tera 100, codéveloppé avec la Direction des applications militaires du CEA (DAM) en 2010, puis le Tera 1000 en 2017 et encore plus en 2023, pour tester et vérifier numériquement certaines théories qui entrent dans le fonctionnement des bombes nucléaires et des missiles. Le programme "Simulation ", géré par la DAM, repose sur les données prises lors des explosions passées. Il repose aussi sur de nouvelles données prises à l'aide d'un laser de très grande puissance, le laser mégaJoule (LMJ) construit récemment à Bordeaux. Celui-ci apporte sur une cible de quelques millimètres, en quelques milliardièmes de seconde, une énergie lumineuse supérieure à un million de joules, afin de mettre la matière dans un état comparable à ce qui se passe dans une bombe atomique.

De même que ses homologues américains et britanniques, respectivement le National Ignition Facility (NIF) et l'Atomic Weapons Establishment (AWE) avec son laser Orion, le centre de Bordeaux (CESTA) est ouvert à la recherche académique. Il dispose d'un laser annexe entièrement civil, PETAL, qui a couté 54 millions d'euros, contre 3 milliards d'euros sur 15 ans pour le LMJ. Son intérêt est d'acquérir notamment des connaissances sur l'interaction laser-matière et sur les plasmas, pour des recherches liées à l'astrophysique, comme les plasmas stellaires, ou à l'énergie civile par fusion. D'après les responsables de la coopération universitaire au NIF et à l'AWE qui ont davantage de recul qu'au LMJ, cette coopération est mutuellement avantageuse [3] : les militaires profitent de regards extérieurs et d'idées nouvelles sur leur installation et sur une petite partie de leur activité, le reste étant "secret défense "; les universitaires peuvent utiliser, mais seulement pour environ 10\% $\mathrm{du}$ temps, les équipements lasers du centre militaire de Bordeaux, qui viennent compléter les lasers de puissance du laboratoire de l'École polytechnique (le LULI) à Palaiseau.

\section{Quel besoin d'une arme nucléaire?}

On peut s'interroger sur la pertinence des technologies duales pour le nucléaire. $\mathrm{Ne}$ serait-il pas bien plus efficace de financer directement les besoins de la recherche civile et de l'industrie dans les domaines du nucléaire (comme c'est le cas par exemple en Allemagne ou au Japon, qui n'ont pas d'armes nucléaires), sans devoir faire appel à des miettes du financement militaire? En outre le nucléaire militaire peut s'arrêter : déjà le rapport du Sénat de 2012 sur "l'avenir des forces nucléaires françaises " [4] disait : " S'il nous fallait dessiner aujourd'hui un format d'armées partant de zéro, il est fort probable que la nécessité d'acquérir une force de frappe nucléaire $[\ldots]$ ne ferait pas partie de nos ambitions de défense. " Le système dual de financement de la recherche ne se justifie que si l'on veut développer un armement nucléaire, ce qui est un choix politique. Or, justement, des raisons politiques militent pour la décroissance et à terme l'arrêt des financements pour l'armement nucléaire, au vu du récent Traité d'interdiction des armes nucléaires ouvert par l'ONU à la signature des États en juillet 2017.

Certains scientifiques, dont nous faisons partie avec le mouvement mondial Pugwash [5], ne souhaitent pas que la recherche nucléaire civile contribue directement à des applications militaires. Ils pensent de plus, avec la Campagne internationale pour l'abolition des armes nucléaires (ICAN), prix Nobel de la Paix 2017, que la dissuasion nucléaire n'est pas une solution durable pour la paix mondiale. Ils sont conscients des risques associés au développement de ces armes, accrus par le risque de prolifération, le piratage informatique, les accidents de maintenance ou de déclenchement de guerre nucléaire par méprise [6], par la production de petites bombes dites "sales » à partir de matériaux radioactifs, etc. Sans même mentionner l'immoralité et l'horreur que représenterait une guerre nucléaire, même limitée !

\section{Références}

1. «Les enjeux industriels et technologiques du renouvellement des deux composantes de la dissuasion ", Rapport Assemblée Nationale $\mathrm{N}^{\circ} 4301$ (décembre 2016).

2. "La nécessaire modernisation de la dissuasion nucléaire ", Rapport Sénat №560 (mai 2017).

3. Voir des exemples de coopérations dans : « Bilan 2015 des publications et de la vie scientifique de la Direction des applications militaires ", Chocs Avancées, 10 (juin 2016).

4. «Rapport d'information sur l'avenir des forces nucléaires françaises ", Rapport Sénat N668 (juillet 2012)

5. J. Bordé, N. Delerue, A. Suzor-Weiner, «Pugwash : les physiciens, l'arme nucléaire, la responsabilité des scientifiques ", Reflets de la Physique 43 (2015) 51-53.

6. Sur les très nombreux accidents technologiques passés ayant impliqué des armements nucléaires et les fausses alertes qui ont failli déclencher des guerres nucléaires, voir, par exemple : J. Villain, Le livre noir du nucléaire militaire, Fayard (2014).

a.Voir dans ce dossier l'article de H. Bercegol (p. 34).

b. Notons que le domaine militaire pousse à développer des systèmes avec des exigences techniques supérieures mais souvent inutiles pour les besoins civils (on n'a pas les mêmes besoins pour la Formule 1 et pour la voiture de tout le monde).

c. On peut lire dans les rapports parlementaires cités en référence que «si la France dispose d'une filière de calcul à haute performance, c'est bien grâce à la dissuasion ". 